\title{
RNA interference as a promising treatment against SARS-CoV-2
}

\author{
Ahmed Donia ${ }^{1}$ (D) $\cdot$ Habib Bokhari ${ }^{1}$
}

Received: 25 August 2020 / Revised: 25 August 2020 / Accepted: 28 August 2020 / Published online: 1 September 2020

(C) Springer Nature Switzerland AG 2020

\begin{abstract}
Until now, there is no current vaccine or treatment against SARS-CoV-2. There are previous successful RNAi studies performed on SARS-CoV. Therefore, similar line of investigation against SARS-CoV-2 could be successful.
\end{abstract}

Keywords SARS-CoV $\cdot$ SARS-CoV-2 $\cdot$ RNAi $\cdot$ Leader sequence

RNA interference (RNAi) is a mechanism through which small interfering RNA (siRNA) triggers post-transcriptional gene silencing. RNAi has shown promising results in the protection from viral invasion, since it inhibits the expression of viral antigens and controls the replication and transcription of the viral genome (Wu and Chan 2006).

Recent comparative genomic analyses of SARS-CoV-2 and SARS-CoV have concluded that the genomic sequences of SARS-CoV and SARS-CoV-2 have extremely high homology at the nucleotide level. Most of SARS-CoV-2 proteins are extremely high homologous (95-100\%) to the proteins of SARS-CoV virus, showing the evolutionary similarity between SARS-CoV and SARS-CoV-2 (Xu et al. 2020). At the whole-genome level, the sequences of SARS-CoV-2 share approximately $79 \%$ sequence identity to SARS-CoV (Zhou et al. 2020).

Previous studies reported that RNAi has shown promising results against SARS-CoV appeared in 2003 (He et al. 2003; Wang et al. 2004; Wu et al. 2005; Li et al. 2005a; Li et al. 2005b; Enjuanes et al. 2001). Since SARS-CoV-2 shares approximately $79 \%$ identity to SARS-CoV, we can expect promising results if researchers explore the similar line of investigation against SARS-CoV-2. Therefore, RNAi could be a promising strategy in SARS-CoV-2 therapy.

Ahmed Donia

ahmeddonia123@yahoo.com

Habib Bokhari

habib@comsats.edu.pk

1 Biosciences Department, Faculty of Science, COMSATS University Islamabad, Islamabad, Pakistan
RNAi (through siRNAs) was successful in targeting the replicase $1 \mathrm{~A}$ region of SARS-CoV, inhibiting SARS-CoV infection in vitro (He et al. 2003). Previous study also reported the production of plasmid-mediated small interfering RNAs (siRNAs) to target the viral RNA polymerase, which successfully inhibited the cytopathic effects of SARS-CoV on Vero cells (Wang et al. 2004). These plasmid-mediated small interfering RNAs also prevented viral replication as seen by titer assays and by calculation of viral RNA and protein levels.

In a previous study, scientists targeted four regions in the SARS-CoV genome: the leader sequence, transcriptionregulating sequence (TRS), 3'-UTR, and the S proteincoding sequence.

They found that $S$ gene specific targeted siRNAs greatly reduced levels of amount of RNA transcripts and viral antigens; although 3'-UTR-oriented siRNAs were not as effective, the two other siRNAs targeting the leader sequence and TRS had no effect (Wu et al. 2005).

Pioneering study found that siRNAs showed prominent prophylactic and therapeutic activity against SARS-CoV in Rhesus macaque (Macaca mulatta) (Li et al. 2005a). These siRNAs were evaluated previously in vitro. To our knowledge, this is the first study to investigate in vivo efficacy of siRNAs against SARS-CoV. The researchers used two siRNA duplexes, siSC2 and siSC5, targeting the SARS-CoV genome at spike protein-coding and ORF1b (NSP12) regions. They found that the siRNAs used provided relief from fever induced by SARS-CoV infection by reducing viral load of SARS-CoV and lowered acute diffuse alveoli damage. The $10-40 \mathrm{mg} / \mathrm{kg}$ accumulated dosages of these siRNAs did not exhibit any sign of siRNA-related toxicity. They found that siRNAs showed anti-SARS-CoV efficacy via either prophylactic or therapeutic regimens. Thus, testing the efficacy of these siRNAs 
(siSC2 and siSC5) against SARS-CoV-2 may also show prominent prophylactic and therapeutic activity.

Based on analysis and comparison of the genomic sequences of SARS-CoV and other coronaviruses, scientists predicted and confirmed a putative $5^{\prime}$ leader sequence with similarity to the conserved coronavirus core leader sequence, $5^{\prime}$-CUAAAC-3', at the $5^{\prime}$-end of the genome (Li et al. 2005b).

The absence of the core CS (5'-CUAAAC- $\left.3^{\prime}\right)$ led to the complete abrogation of mRNA transcription of transmissible gastroenteritis coronavirus (TGEV), which is a coronavirus that infects pigs (Enjuanes et al. 2001). Replication of coronavirus occurs through a unique mechanism using leader sequence in the transcripts when starting transcription from the coronavirus genome. The genomic and the subgenomic mRNAs of coronaviruses have an identical 5 ' leader sequence (via a unique mechanism called discontinuous transcription) and common 3 '-ends, a unique feature in coronavirus replication.

Leader sequence was found to be highly conserved between different strains of SARS-CoV. Transcriptionregulating sequences (TRSs) found at the $5^{\prime}$-end of each gene are considered to be signals that regulate the discontinuous transcription of subgenomic mRNAs. TRSs have a conserved 6-7 nucleotide core sequence surrounded by variable sequences. The leader sequence and the TRSs have pivotal roles in the gene expression of coronavirus during the replication process (Enjuanes et al. 2001). The siRNA targeting the leader sequence reduced the abundance of mRNA and protein expression levels of the reporter genes in $293 \mathrm{~T}$ cell line. They also found that the siRNA targeting the leader sequence could inhibit the replication of SARS-CoV via silencing gene expression in Vero E6 cells. Additionally, siRNA targeting the leader sequence exhibited a much potent inhibitory effect on the replication of SARS-CoV than the siRNAs targeting the spike gene made (Enjuanes et al. 2001). The identical 5' leader sequence and conserved nucleotides seen in TRSs may be considered as an ideal target using RNAi against SARSCoV-2.

Keeping in mind that genomic sequences of SARS-CoV and SARS-CoV-2 exhibit extremely high homology at the nucleotide level and there is a previous successful studies of RNAi against SARS-CoV, there is an urgent need to investigate the efficacy of RNAi against SARS-CoV-2.

\section{Compliance with ethical standards}

Conflict of interest The authors declare that they have no conflict of interest.

\section{References}

Enjuanes L, Sola I, Almazan F, Ortego J, Izeta A, Gonzalez JM, Alonso S, Sanchez JM, Escors D, Calvo E, Riquelme C (2001) Coronavirus derived expression systems. Journal of biotechnology. 88(3):183204

He ML, Zheng B, Peng Y, Peiris JS, Poon LL, Yuen KY, Lin MC, Kung HF, Guan Y (2003) Inhibition of SARS-associated coronavirus infection and replication by RNA interference. Jama. 290(20):26652666

Li BJ, Tang Q, Cheng D, Qin C, Xie FY, Wei Q, Xu J, Liu Y, Zheng BJ, Woodle MC, Zhong N (2005a) Using siRNA in prophylactic and therapeutic regimens against SARS coronavirus in Rhesus macaque. Nature medicine. 11(9):944-951

Li T, Zhang Y, Fu L, Yu C, Li X, Li Y, Zhang X, Rong Z, Wang Y, Ning H, Liang R (2005b) siRNA targeting the leader sequence of SARSCoV inhibits virus replication. Gene Therapy. 12(9):751-761

Wang Z, Ren L, Zhao X, Hung T, Meng A, Wang J, Chen YG (2004) Inhibition of severe acute respiratory syndrome virus replication by small interfering RNAs in mammalian cells. Journal of virology. 78(14):7523-7527

Wu CJ, Chan YL (2006) Antiviral applications of RNAi for coronavirus. Expert opinion on investigational drugs. 15(2):89-97

Wu CJ, Huang HW, Liu CY, Hong CF, Chan YL (2005) Inhibition of SARS-CoV replication by siRNA. Antiviral research. 65(1):45-48

Xu J, Zhao S, Teng T, Abdalla AE, Zhu W, Xie L, Wang Y, Guo X (2020) Systematic comparison of two animal-to-human transmitted human coronaviruses: SARS-CoV-2 and SARS-CoV. Viruses. 12(2):244

Zhou P, Yang XL, Wang XG, Hu B, Zhang L, Zhang W, Si HR, Zhu Y, Li B, Huang CL, Chen HD (2020) A pneumonia outbreak associated with a new coronavirus of probable bat origin. Nature 579(7798): $270-273$

Publisher's note Springer Nature remains neutral with regard to jurisdictional claims in published maps and institutional affiliations. 